\title{
Corrections to \\ "Bifurcation from a saddle connection in functional differential equations: An approach with inclination lemmas"
}

(Dissertationes Math. 291 (1990))

\author{
by Hans-Otto Walther (München)
}

Page 21, line 16: Delete "and is continuous".

Include "Each map $X(t, \cdot, a): C \rightarrow C$ is of class $C^{1}$."

Page 31, line 10: Add a line:

"(vi) The maps $D G, D G^{-}, D_{1} G, D_{1} G^{-}$are bounded."

Page 32, lines 14 and 15: Delete "and are continuous".

Add "Each map $Y(t, \cdot, a), R(t, \cdot, a)$ is of class $C^{1}$."

Page 32, lines 24-26: Delete "The assignments ... into $L_{c}(C, C)$."

Page 33, lines 10-14: Replace these lines by the following text.

"Proposition 5.! There exists a constant const $\geq 0$ such that we have

$$
\begin{aligned}
\left|D_{2} R_{p_{a}}(t, \psi)\right|+\left|D_{2} R_{q_{a}}(t, \psi)\right| & <\text { const } \\
& \text { for all }(t, \psi, a) \in[0, N] \times D^{1} \times A_{7} .
\end{aligned}
$$

Pr o of. Let $(t, \psi, a) \in[0, N] \times D^{1} \times A_{7}$ be given. We have

$$
\left|D_{2} R(t, \psi, a)\right| \leq\left|D_{2} Y(t, \psi, a)\right|+|T(t, \cdot, a)|
$$

and

$$
\begin{aligned}
\left|D_{2} Y(t, \psi, a)\right| & \leq \sup \left|D_{1} G\right|\left|D_{2} X\left(t, G^{-}(\psi, a), a\right)\right| \sup \left|D^{1} G^{-}\right| \\
& \leq \sup \left|D_{1} G\right| \sup \left|D_{1} G^{-}\right|\left(1+\max \left|h^{\prime}\right|\right)^{N+1},
\end{aligned}
$$

by Proposition 5.1(vi) and Corollary 3.1. There is a constant $k_{00} \geq 1$ such that

$$
|T(t, \cdot, a)| \leq k_{00} e^{-\lambda t} \quad \text { for all } t \geq 0, a \in A_{7} \subset A_{3} .
$$

Now the desired estimate becomes obvious. 
From Proposition 5.3(i) we infer that there exist an open ball $D^{2.1} \subset D^{1}$, centered at $0 \in C$, and an open interval $A_{8}$ (with $\mathrm{cl} A_{8} \subset A_{7}$ ) such that we have"

$(5.7) \ldots$

Page 34, lines 25 and 27: Replace "c" by "const".

Page 34, line 28: Replace " $\left(1+c+k_{0}\right) e^{-\lambda_{1} N "}$ by " $\left(1+\right.$ const $\left.+k_{0}\right) e^{-\lambda_{1} N "}$.

Page 35, lines 10 and 11: Write

$$
\text { " }\left|p_{a} \circ Y_{a}(3, \psi)\right| \leq(\text { const }+1) e^{3 \mu_{2}}\left|p_{a} \psi\right| " .
$$

Page 35, line 21: Write

$$
\text { " } c_{4}:=\frac{c_{3}}{(\text { const }+1) e^{3 \mu_{2}}} " .
$$

Page 36, lines 25 and 26: Replace "c" by "const".

MATHEMATISCHES INSTITUT

LUDWIG-MAXIMILIANS -UNIVERSITÄT

THERESIENSTR. 39

D-8000 MÜNCHEN 2, GERMANY

Reçu par la Rédaction le 12.11.1992 